PROCEEDINGS OF THE

AMERICAN MATHEMATICAL SOCIETY

Volume 127, Number 9, Pages 2601-2609

S 0002-9939(99)04880-7

Article electronically published on April 15, 1999

\title{
ON THE EXISTENCE OF MAXIMAL COHEN-MACAULAY MODULES OVER $p$ th ROOT EXTENSIONS
}

\author{
DANIEL KATZ
}

(Communicated by Wolmer V. Vasconcelos)

\begin{abstract}
Let $S$ be an unramified regular local ring having mixed characteristic $p>0$ and $R$ the integral closure of $S$ in a $p$ th root extension of its quotient field. We show that $R$ admits a finite, birational module $M$ such that $\operatorname{depth}(M)=\operatorname{dim}(R)$. In other words, $R$ admits a maximal Cohen-Macaulay module.
\end{abstract}

\section{INTRODUCTION}

Let $R$ be a Noetherian local ring. In considering the local homological conjectures over $R$, one may reduce to the situation where $R$ is a finite extension of an unramified regular local ring $S$. Therefore, it is a natural point of departure to assume that $R$ is the integral closure of $S$ in a "well-behaved" algebraic extension of its quotient field. Certainly, when $S$ has mixed characteristic $p>0$, one ought to consider the case that $R$ is the integral closure of $S$ in an extension of its quotient field obtained by adjoining the $p$ th root of an element of $S$. This was done in [Ko] where it was shown that $S$ is a direct summand of $R$, i.e., the Direct Summand Conjecture holds for the extension $S \subseteq R$. In this note we show that a number of the other local homological conjectures hold for such $R$ by showing that $R$ admits a finite, birational module $M$ satisfying $\operatorname{depth}(M)=\operatorname{dim}(R)$ (see $[\mathrm{H}]$ ). In other words, $R$ admits a maximal Cohen-Macaulay module. Such a module is necessarily free over $S$. Aside from regularity, one of the crucial points in the mixed characteristic case seems to be that $S / p S$ is integrally closed. By contrast, using an example from $[\mathrm{HM}]$, Roberts has noted that even if $S$ is a Cohen-Macaulay UFD and $R$ is the integral closure of $S$ in a quadratic extension of quotient fields, $R$ needn't admit a finite, $S$-free module at all (see [R]). For the example in question, $S$ has mixed characteristic 2 , yet $S / 2 S$ is not integrally closed.

\section{Preliminaries}

In this section we will establish our notation and present a few preliminary observations. Throughout, $S$ will be a Noetherian normal domain with quotient field $L$. We assume $\operatorname{char}(L)=0$. Fix $p \in \mathbb{Z}$ to be a prime integer and suppose that either $p$ is a unit in $S$ or that $p S$ is a (proper) prime ideal and $S / p S$ is integrally closed. Let $f \in S$ be an element that is not a $p$ th power and select $W$ an indeterminate. Write $F(W):=W^{p}-f \in S[W]$, a monic irreducible polynomial

Received by the editors August 26, 1997 and, in revised form, November 26, 1997.

1991 Mathematics Subject Classification. Primary 13B21, 13B22, 13H05, 13 H15. 
and let $R$ denote the integral closure of $S$ in $K:=L(\omega)$, for $\omega$ a root of $F(W)$. Thus $R$ is the integral closure of $S[\omega]$.

Our strategy in this paper is to exploit the fact that $R$ can be realized as $J^{-1}$ for a suitable ideal $J \subseteq S[\omega]$. The study of birational algebras of the form $J^{-1}$ seems to have captured the attention of a number of researchers during the last few years, albeit in notably different contexts (see [EU], [Ka], [KU], [MP] and [V]). Since $J^{-1}$ inherits $S_{2}$ from $S[\omega]$, this means that in attempting to "construct" $R$, if the candidate is $J^{-1}$ for some $J$, then only the condition $R_{1}$ must be checked.

The following proposition summarizes some of the conditions relating $R$ to $J^{-1}$ for suitable $J$ that we will call upon in the next section. Parts (i) and (ii) of the proposition were inspired by the main results in $[\mathrm{V}]$ and Proposition 3.1 in $[\mathrm{KU}]$. Special cases of part (iii) of the proposition have apparently been known to algebraic geometers for a long while. For some historical comments and fascinating variations, the interested reader should consult $[\mathrm{KU}]$.

Proposition 2.1. Let $A$ be a Noetherian domain satisfying $S_{2}$ and assume that $A^{\prime}$, the integral closure of $A$, is a finite A-module.

(i) Suppose $\left\{P_{1}, \ldots, P_{n}\right\}$ are the height one primes of $A$ for which $A_{P_{i}}$ is not a $D V R$. If for each $1 \leq i \leq n, \operatorname{rad}\left(J_{i}\right)=P_{i}$ and $\left(J_{i}^{-1}\right)_{P_{i}}=A_{P_{i}}^{\prime}$, then $A^{\prime}=J^{-1}$, for $J:=J_{1} \cap \cdots \cap J_{n}$.

(ii) If $A \neq A^{\prime}$, then $A^{\prime}=J^{-1}$, for some height one unmixed ideal $J \subseteq A$. Moreover, if $A$ is Gorenstein in codimension one, then $A^{\prime}=J^{-1}$ for a unique height one unmixed ideal $J$ satisfying $J \cdot J^{-1}=J=\left(J^{-1}\right)^{-1}$.

(iii) Suppose that $A=B /(F)$ for $F \in B$ a principal prime and $\tilde{J} \subseteq B$ is a grade two ideal arising as the ideal of $n \times n$ minors of an $(n+1) \times n$ matrix $\phi$. Assume further that $F \in \tilde{J}$ and set $J:=\tilde{J} /(F)$. Let $\Delta_{1}, \ldots, \Delta_{n+1}$ denote the signed minors of $\phi$, write $F:=b_{1} \Delta_{1}+\cdots+b_{n+1} \Delta_{n+1}$ and let $\phi^{\prime}$ denote the $(n+1) \times(n+1)$ matrix obtained by augmenting the column of $b_{i}^{\prime} s$ to $\phi$ (so $F$ is the determinant of $\left.\phi^{\prime}\right)$. Then $J^{-1}$ can be generated as an $A$-module by $\left\{\psi_{1,1} / \delta_{1}, \ldots, \psi_{n+1, n+1} / \delta_{n+1}=1\right\}$, where $\psi_{i, i}$ denotes the image in $A$ of the $(i, i)$ th cofactor of $\phi^{\prime}$ and $\delta_{i}$ denotes the image of $\Delta_{i}$ in $A$ (which we assume to be non-zero). Moreover, $p . d \cdot{ }_{B}(J)=p \cdot d \cdot{ }_{B}\left(J^{-1}\right)=1$.

Proof. To prove (i), note that $J_{Q}^{-1}=A_{Q}^{\prime}$ for all height one primes $Q \subseteq A$. Since $J^{-1}$ and $A^{\prime}$ are birational and satisfy $S_{2}$, we obtain $J^{-1}=A^{\prime}$. For the first statement in (ii), we may, by part (i), consider the case where $A$ is a one-dimensional local ring which is not a DVR. Let $Q$ denote the maximal ideal of $A$. Then $Q Q^{-1} \subseteq Q$. Since it always holds that $Q \subseteq Q Q^{-1}$, we have $Q=Q Q^{-1}$. Therefore $Q^{-1}$ is a finite ring extension properly containing $A$ (since for any ideal $J,\left(J J^{-1}\right)^{-1}$ is a ring). If $Q^{-1}=A^{\prime}$, we're done. If not, then since $Q^{-1}$ inherits $S_{2}$ from $A, Q^{-1}$ contains a height one prime $P$ for which $\left(Q^{-1}\right)_{P}$ is not a DVR. Thus $P^{-1}$ is a finite ring extension properly containing $Q^{-1}$. An easy calculation shows that $P^{-1}$, considered over $Q^{-1}$, equals $(Q P)^{-1}$, considered over $A$. Iterating this process shows we eventually obtain $A^{\prime}=J^{-1}$, for some $J \subseteq A$. Now suppose that $A$ is Gorenstein in codimension one. Then $I_{Q}=\left(\left(I^{-1}\right)^{-1}\right)_{Q}$, for all ideals $I \subseteq A$ and all height one primes $Q \subseteq A$. Therefore, $I=\left(I^{-1}\right)^{-1}$, for all height one, unmixed ideals $I \subseteq A$. In particular, this holds for $J$. Moreover, if $J^{-1}=A^{\prime}=K^{-1}$, for $K$ height one and unmixed, then $J=K$. Finally, since $J^{-1}$ is a ring, $\left(J \cdot J^{-1}\right) \cdot J^{-1}=J \cdot J^{-1}$, so $J \cdot J^{-1} \subseteq\left(J^{-1}\right)^{-1}=J$. Thus, $J \cdot J^{-1}=J$, as desired. For (iii), the description of 
the generators for $J^{-1}$ follows either from [MP], Proposition 3.14 or [KU], Lemma 2.5. For the second part of (iii), see [KU], Proposition 3.1.

Returning to our basic set-up, we note that since $S$ is a normal domain, $S[\omega]$ satisfies Serre's condition $S_{2}$. Moreover, since $\operatorname{char}(S)=0, R$ is a finite $S$-module. Thus Proposition 2.1 applies. In Section 3 we will identify the ideal $J \subseteq S[\omega]$ for which $J^{-1}=R$. In the meantime, we observe that if $p$ is not a unit in $S$, then there is a unique height one prime in $S[\omega]$ containing $p$. Suppose $p \mid f$. Then $P:=(\omega, p)$ is clearly the unique height one prime in $S[\omega]$ containing $p$. Moreover, $S[\omega]_{P}$ is a DVR if and only if $p^{2} \nmid f$. Suppose $p \nmid f$. If $f$ is not a $p$ th power modulo $p S$, then $f$ is not a $p$ th power over the quotient field of $S / p S$ (since $S / p S$ is integrally closed) and it follows that $F(W)$ is irreducible $\bmod p S$. Thus $(p, F(W))$ is the unique height two prime in $S[W]$ containing $F(W)$ and $p$, so $p S[\omega]$ is the unique height one prime in $S[\omega]$ containing $p$. If $f \equiv h^{p} \bmod p S$, then $F(W) \equiv(W-h)^{p} \bmod p S$ and it follows that $(\omega-h, p) S[\omega]$ is the unique height one prime in $S[\omega]$ containing $p$. Thus, in all cases, there exists a unique height one prime in $S[\omega]$ lying over $p S$. For the remainder of the paper, we call this prime $P$. Suppose $f=h^{p}+g p$, so $P=(\omega-h, p) S[\omega]$. Write $\tilde{P}:=(W-h, p) S[W]$ for the preimage of $P$ in $S[W]$. Then

$$
F(W)=W^{p}-h^{p}-g p=\left(W^{p-1}+\cdots+h^{p-1}\right) \cdot(W-h)-g p .
$$

In $S[W], W^{p-1}+\cdots+h^{p-1} \equiv p h^{p-1} \operatorname{modulo}(W-h)$, so $W^{p-1}+\cdots+h^{p-1} \in \tilde{P}$. Thus, $F(W) \in \tilde{P}^{2}$ if and only if $p \mid g$. In other words, in all cases, $P_{P}$ is not principal if and only if $f=h^{p}+p^{2} g$, for some $h, g \in S$.

\section{The MAIN RESUlT}

In this section we will present our main result, Theorem 3.8. Lemmas 3.2 and 3.3 will enable us to describe the ideal $J \subseteq S[\omega]$ for which $R=J^{-1}$. We will then see in the proof of Theorem 3.8 that the module we seek has the form $I^{-1}$, for some ideal $I \subseteq J$.

Lemma 3.1. Suppose $p$ is not a unit in $S, h \in S \backslash p S$ and $p=2 k+1$. Set

$$
C:=\sum_{j=1}^{k}(-1)^{j+1}\left(\begin{array}{l}
p \\
j
\end{array}\right)(W \cdot h)^{j}\left[W^{p-2 j}-h^{p-2 j}\right],
$$

$C^{\prime}:=C \cdot(p(W-h))^{-1}$ and $\tilde{P}:=(p, W-h) \cdot S[W]$. Then $C^{\prime} \notin \tilde{P}$.

Proof. Note that since $p$ divides $\left(\begin{array}{c}p \\ j\end{array}\right)$ for all $1 \leq j \leq k, C^{\prime}$ is a well-defined element of $S[W]$. Now, $C^{\prime} \notin \tilde{P}$ if and only if the residue class of $C^{\prime}$ modulo $W-h$, as an element of $S$, does not belong to $p S$ if and only if $\sum_{j=1}^{k}(-1)^{j+1}\left(\begin{array}{l}p \\ j\end{array}\right) \frac{h^{p-1}}{p}(p-2 j)$, as an element of $S$, is not divisible by $p$. Since

$$
\sum_{j=1}^{k}(-1)^{j+1}\left(\begin{array}{l}
p \\
j
\end{array}\right) h^{p-1}
$$

is divisible by $p$ and $h^{p-1}$ is not divisible by $p$, it is enough to show that

$$
\sum_{j=1}^{k}(-1)^{j+1}\left(\begin{array}{l}
p \\
j
\end{array}\right) \frac{2 j}{p}
$$


is not divisible by $p$, as an element of $S$. However,

$$
\sum_{j=1}^{k}(-1)^{j+1}\left(\begin{array}{l}
p \\
j
\end{array}\right) \frac{2 j}{p}=2 \cdot \sum_{j=1}^{k}(-1)^{j+1}\left(\begin{array}{c}
p-1 \\
j-1
\end{array}\right)=(-1)^{k+1}\left(\begin{array}{c}
2 k \\
k
\end{array}\right) .
$$

Because $p$ does not divide $\left(\begin{array}{c}2 k \\ k\end{array}\right)$ in $\mathbb{Z}, p$ does not divide $\left(\begin{array}{c}2 k \\ k\end{array}\right)$ as an element of $S$ (since $p S \neq S$ ). Thus $C^{\prime} \notin \tilde{P}$, as claimed.

For the next lemma, we borrow the following terminology from [Kap]. We shall say that $f \in S$ is "square-free" if $q S_{q}=f S_{q}$ for all height one prime ideals $q \subseteq S$ containing $f$. Since $F^{\prime}(\omega) \cdot R \subseteq S[\omega]$ and $\omega \cdot F^{\prime}(\omega)=p \cdot f$, it follows from the discussion in Section 2 that if $f$ is square-free, then either $R=S[\omega]$ or $P$ is the only height one prime for which $S[\omega]_{P}$ is not a DVR.

Lemma 3.2. Suppose $f \in S$ is square-free and $S[\omega] \neq R$ (thus $p$ is not a unit in $S)$. Then $R=P^{-1}$. Moreover, $R$ is a free $S$-module.

Proof. We first consider the case $p>2$. Since $S[\omega]$ is not integrally closed, we have $f=h^{p}+p^{2} g$, for some $h$ not divisible by $p$ and $g \neq 0$ in $S$. Thus, $P=(\omega-h, p) S[\omega]$. It follows from the proof and statement of Proposition 2.1 that $P^{-1}$ is a ring and that $P^{-1}$ is generated as an $S[\omega]$-module by $\{1, \tau\}$, for

$$
\tau=\frac{1}{p} \cdot \sum_{j=1}^{p} \omega^{p-j} h^{j-1}=\frac{g \cdot p}{\omega-h} .
$$

Therefore $P^{-1}=S[\omega, \tau]$. If we show that $S[\omega, \tau]$ satisfies $R_{1}$, then $S[\omega, \tau]=R$, since $P^{-1}$ satisfies $S_{2}$ (as an $S[\omega]$-module and as a ring). Since $f$ is square-free, it suffices to show that $P_{Q}^{-1}$ is a DVR for each height one $Q \subseteq P^{-1}$ containing $p$. To do this, we find an equation satisfied by $\tau$ over $S[\omega]$. On the one hand,

$$
(\omega-h) \cdot \tau=0 \cdot(w-h)+g \cdot p .
$$

On the other hand,

$$
p \cdot \tau=(\omega-h)^{p-2} \cdot(\omega-h)+c^{\prime} \cdot p,
$$

where $c^{\prime}$ denotes the image in $S[\omega]$ of the element $C^{\prime} \in S[W]$ defined in Lemma 3.1. Therefore, by the standard determinant argument, $\tau$ satisfies

$$
l(T):=T^{2}-c^{\prime} T-g(\omega-h)^{p-2}
$$

over $S[\omega]$. Now, let $\pi: S[W, T] \rightarrow S[\omega, \tau]$ denote the canonical map and set $H:=\operatorname{ker}(\pi)$ and let $Q \subseteq S[\omega, \tau]$ be any height one prime containing $p$. Then $Q$ corresponds to a height three prime $Q^{\prime} \subseteq S[W, T]$ containing $p$ and $H$. Since $P \subseteq Q$ and $H \subseteq Q^{\prime}, W-h$ and $T^{2}-C^{\prime} T-g(W-h)^{p-2}$ belong to $Q^{\prime}$. Therefore, $Q^{\prime}=(p, W-h, T)$ or $Q^{\prime}=\left(p, W-h, T-C^{\prime}\right)$. Suppose $Q^{\prime}=(p, W-h, T)$. Then $Q=(p, \omega-h, \tau) S[\omega, \tau]$. We have

$$
\tau^{2}-c^{\prime} \tau-g(\omega-h)^{p-2}=0 \quad \text { and } \quad p\left(\tau-c^{\prime}\right)=(\omega-h)^{p-1} .
$$

By Lemma 3.1, $c^{\prime} \notin Q$, so $\tau-c^{\prime} \notin Q$, and it follows that $Q_{Q}=(\omega-h)_{Q}$. Now suppose $Q^{\prime}=\left(p, W-h, T-C^{\prime}\right)$. Then $Q=\left(p, \omega-h, \tau-c^{\prime}\right) S[\omega, \tau]$. Since

$$
\tau^{2}-c^{\prime} \tau-g(\omega-h)^{p-2}=0 \quad \text { and } \quad(\omega-h) \cdot \tau=g \cdot p,
$$

it follows that $Q_{Q}=(p)_{Q}$ (since $\tau \notin Q$, by Lemma 3.1). Thus, in either case, $Q_{Q}$ is principal, so $R=S[\omega, \tau]=P^{-1}$. 
The proof is similar if $p=2$ and $f=h^{2}+4 g$, with $2 \nmid h$. One notes that $P^{-1}=S[\omega, \tau]=S[\tau]$, for $\tau:=\frac{h+\omega}{2}$ and that $\tau$ satisfies $l(T):=T^{2}-h T-g$. To show $R=S[\tau]$, one uses the fact that $l(T)$ and $l^{\prime}(T)$ are relatively prime over the quotient field of $S / 2 S$.

To see that $R$ is a free $S$-module, we first note that $R$ is clearly generated as an $S$-module by the set $\left\{1, \omega, \ldots, \omega^{p-1}, \tau, \tau \omega, \ldots, \tau \omega^{p-1}\right\}$. However, $\tau \omega=p g \cdot 1+h \cdot \tau$. This implies that $\tau \omega^{i}$ belongs to the $S$-module generated by $\left\{1, \omega, \ldots, \omega^{p-1}, \tau\right\}$, for all $1 \leq i \leq p-1$. Moreover, since

$$
\omega^{p-1}=-h^{p-1} \cdot 1-h^{p-2} \cdot \omega-\cdots-h \cdot \omega^{p-2}+p \cdot \tau,
$$

we may dispose of $\omega^{p-1}$ as well. Thus, $R$ is generated as an $S$-module by the set $\left\{1, \omega, \ldots, \omega^{p-2}, \tau\right\}$. Since these elements are clearly linearly independent over $S$, $R$ is a free $S$-module.

Lemma 3.3. Suppose $f=\lambda a^{e}$, with $a \in S$ a prime element, $\lambda$ a unit in $S$ and $2 \leq e<p$. If $p$ is not a unit in $S$, assume $a=p$. Then there exist integers $1 \leq s_{1}<s_{2}<\cdots<s_{e-1}<p$ satisfying

(i) $s_{e-i} \leq p-s_{i}, 1 \leq i \leq e-1$.

(ii) $R=J^{-1}$ for $J:=\left(\omega^{s_{e-1}}, \omega^{s_{e-2}} a, \ldots, \omega^{s_{1}} a^{e-2}, a^{e-1}\right) S[\omega]$.

Proof. We begin by noting that either condition in the hypothesis implies that $Q:=(\omega, a) S[\omega]$ is the only height one prime for which $S[\omega]_{Q}$ is not a DVR. Now, since $p$ and $e$ are relatively prime, we can find positive integers $u$ and $v$ such that $1=u \cdot p+(-v) \cdot e$. If we set $\tau:=\frac{a^{u}}{\omega^{v}}$, then $\tau^{e}=\lambda^{-u} \omega$ and $\tau^{p}=\lambda^{-v} a$. It follows that $S[\omega, \tau]=S[\tau]=R$, since either $p$ is a unit and $a$ is square-free or $p$ is not a unit and $(\tau, p) S[\tau]=\tau S[\tau]$. Thus, $\left\{1, \tau, \ldots, \tau^{e-1}\right\}$ generate $R$ as an $S[\omega]$ module. Since $u$ and $e$ are relatively prime, the set $\{u j\}_{1 \leq j \leq e-1}$, when reduced $\bmod e$, equals the set $\{i\}_{1 \leq i \leq e-1}$. This will enable us to replace the generators $\left\{1, \tau, \ldots, \tau^{e-1}\right\}$ by $\left\{1, \frac{\lambda a}{\omega^{s_{1}}}, \ldots, \frac{\lambda a^{e-1}}{\omega^{s^{e-1}}}\right\}$. To elaborate, given $1 \leq i \leq e-1$, there is a unique $1 \leq j_{i} \leq e-1$ such that $u j_{i} \equiv i(\bmod e)$. Write $u j_{i}=t_{i} e+i, t_{i} \geq 0$. Then

$$
(1+v e) j_{i}=p u j_{i}=t_{i} e p+i p
$$

so $\left(v j_{i}\right) e+j_{i}=\left(t_{i} p\right) e+i p$. If we write $i p=s_{i} e+r$, with $0 \leq r<e$, then uniqueness of the euclidean algorithm gives $v j_{i}=t_{i} p+s_{i}$ and $r=j_{i}$. Thus, $\tau^{j_{i}}=\frac{a^{u j_{i}}}{\omega^{v j_{i}}}=\frac{a^{i}}{\lambda^{t_{i}} \omega^{s_{i}}}$ and $i p=s_{i} e+j_{i}$. For $i=e-1$, this yields $s_{e-1}<p$. Moreover, $p=\left(s_{i+1}-s_{i}\right) e+\left(j_{i+1}-j_{i}\right)$, so $s_{i+1}-s_{i}>0$. Similarly, $e p=\left(s_{e-i}+s_{i}\right) e+\left(j_{e-i}+j_{i}\right)$, so $s_{e-i}+s_{i} \leq p$. Thus, $s_{1}, \ldots, s_{e-1}$ have the required numerical properties.

We now have $\left\{1, \tau, \ldots, \tau^{e-1}\right\}=\left\{1, \frac{a}{\lambda^{t_{1} \omega^{s_{1}}}}, \ldots, \frac{a^{e-1}}{\lambda^{e_{e-1} \omega^{s_{e-1}}}}\right\}$. Multiplying by appropriate powers of $\lambda$ allows us to use $\left\{1, \frac{\lambda a}{\omega^{s_{1}}}, \ldots, \frac{\lambda a^{e-1}}{\omega^{s^{e-1}}}\right\}$ as a generating set for $R$ over $S[\omega]$. In Proposition 2.1 take $A:=S[\omega], B:=S[W], F:=F(W)$ and $\tilde{J}$ the ideal of $(e-1) \times(e-1)$ signed minors of the $e \times(e-1)$ matrix

$$
\phi=\left(\begin{array}{ccccc}
-a & 0 & \cdots & 0 & 0 \\
W^{\alpha_{e-1}} & -a & \cdots & 0 & 0 \\
0 & W^{\alpha_{e-2}} & \cdots & 0 & 0 \\
\vdots & \vdots & \ddots & \vdots & \vdots \\
0 & 0 & \cdots & W^{\alpha_{2}} & -a \\
0 & 0 & \cdots & 0 & W^{\alpha_{1}}
\end{array}\right)
$$


with $\alpha_{1}+\alpha_{2}+\cdots+\alpha_{i}=s_{i}$, for $1 \leq i \leq e-1$. To obtain $\phi^{\prime}$, we augment $\phi$ by the column whose transpose is $\left(W^{p-c}, 0, \ldots, 0,(-1)^{e} \lambda a\right)\left(\operatorname{so} \operatorname{det}\left(\phi^{\prime}\right)=F(W)\right)$. Then $J^{-1}$ is generated as an $S[\omega]$-module by $\left\{1, \frac{\lambda a}{\omega^{s_{1}}}, \ldots, \frac{\lambda a^{e-1}}{\omega^{s e-1}}\right\}$. Thus, $R=S[\omega, \tau]=$ $J^{-1}$ for $J=\left(\omega^{s_{e-1}}, \omega^{s_{e-2}} a, \ldots, a^{e-1}\right)$, as desired.

For a proof of the next lemma, see [Ka], Lemma 4.1.

Lemma 3.4. In $S[W]$ consider the ideals $H:=\left(W^{e_{k}}, W^{e_{k-1}} a_{1}, \ldots, W^{e_{1}} a_{k-1}, a_{k}\right)$ and $K:=\left(W^{f_{t}}, W^{f_{t-1}} b_{1}, \ldots, W^{f_{1}} b_{t-1}, b_{t}\right)$, where

(i) $e_{k}>e_{k-1}>\cdots>e_{1}$ and $f_{t}>f_{t-1}>\cdots>f_{1}$.

(ii) $a_{1}\left|a_{2}\right| \cdots \mid a_{k}$ and $b_{1}\left|b_{2}\right| \cdots \mid b_{t}$.

(iii) Each $a_{i}$ and $b_{j}$ is a product of prime elements.

(iv) For all $i$ and $j, a_{i}$ and $b_{j}$ have no prime factor in common.

Then there exist integers $g_{s}>\cdots>g_{1}$ and products of primes $c_{1}\left|c_{2}\right| \cdots \mid c_{s}$ such that $H \cap K=\left(W^{g_{s}}, W^{g_{s-1}} c_{1}, \ldots, W^{g_{1}} c_{s-1}, c_{s}\right)$. Moreover, $H, K$ and $H \cap K$ are all grade two perfect ideals.

Lemma 3.5. Let $A$ be a domain and $I \subseteq J$ ideals such that $J^{-1}$ is a ring. Then $I^{-1}$ is a $J^{-1}$-module if and only if $I^{-1}=\left(I \cdot J^{-1}\right)^{-1}$. In particular, if $x \in J$ and $x \cdot J^{-1} \subseteq J$, then $\left(x \cdot J^{-1}\right)^{-1}$ is a $J^{-1}$-module.

Proof. We first observe $\left(I \cdot J^{-1}\right)^{-1}$ is always a $J^{-1}$-module. Indeed, $y \in\left(I \cdot J^{-1}\right)^{-1}$ implies $I \cdot J^{-1} y \subseteq R$. Thus $J^{-1} J^{-1} y=J^{-1} y \subseteq I^{-1}$, so $\left(I \cdot J^{-1}\right)\left(J^{-1} y\right) \subseteq R$ and $J^{-1} y \subseteq\left(I \cdot J^{-1}\right)^{-1}$. Therefore, $\left(I \cdot J^{-1}\right)^{-1}$ is a $J^{-1}$-module and the first statement follows easily from this. For the second statement, we note that if $x \cdot J^{-1} \subseteq J$, then for $I:=x \cdot J^{-1}, I \cdot J^{-1}=x \cdot J^{-1} J^{-1}=x \cdot J^{-1}=I$. Thus, $I^{-1}=\left(I \cdot J^{-1}\right)^{-1}$, so $I^{-1}$ is a $J^{-1}$-module by the first statement.

Remark 3.6. Proposition 2.2 in [Ko] states that $R$ is a free $S$-module, if $S$ is an unramified regular local ring and $p \mid f$. The proof shows that $R$ is a free $S$-module just under the assumption that $f$ can be written as a product of primes and $S / p S$ is a domain. In [Ko], Proposition 1.5, it is shown that if $S$ is a UFD, then there exists a free $S$-module $F \subseteq R$ such that $p R$ is contained in $F$. Thus, if $p$ is a unit in $S$, then $R$ is also a free $S$-module. Finally, if $f$ is square-free, $R$ is a free $S$-module by Lemma 3.2. We record these facts in a common setting in the following proposition. For a version of the proposition for $p^{n}$ th root extensions, see [Ka], Theorem 4.2.

Proposition 3.7. In addition to our standing hypotheses, assume that $S$ is a UFD. Then $R$ is a free $S$-module in each of the following cases:

(i) $p$ is a unit in $S$.

(ii) $p$ is not a unit and either $p \mid f$ or $f$ is square-free.

We are now ready for our theorem.

Theorem 3.8. Assume that $S$ is a regular local ring. Then there exists a finite, birational $R$-module $M$ satisfying depth ${ }_{S}(M)=\operatorname{dim}(R)$. In other words, $M$ is a maximal Cohen-Macaulay module for $R$.

Proof. By Proposition 3.7, $R$ is a free $S$-module, and therefore Cohen-Macaulay, unless we assume that $p$ is not a unit in $S, p \nmid f$ and $f$ is not square-free. In particular, we may assume that $f$ is not a unit in $S$. Factor $f$ as a unit $\lambda$ times prime elements $a_{i}$, say $f=\lambda a_{1}^{e_{1}} \cdots a_{r}^{e_{r}}$. We may assume that for $1 \leq t \leq r, 1<e_{i}<p$, if $1 \leq i \leq t$ and $e_{i}=1$, if $t<i \leq r$. Set $Q_{i}:=\left(\omega, a_{i}\right) S[\omega]$ for $1 \leq i \leq t$. For 
each $1 \leq i \leq t$ choose $s(i, 1)<\cdots<s\left(i, e_{i}-1\right)$ satisfying the conclusion of Lemma 3.3 over $S[\omega]_{Q_{i}}$ and set $J_{i}:=\left(\omega^{s\left(i, e_{i}-1\right)}, \omega^{s\left(i, e_{i}-2\right)} a_{i}, \ldots, \omega^{s(i, 1)} a_{i}^{e_{i}-2}, a_{i}^{e_{i}-1}\right) S[\omega]$. Thus, $R_{Q_{i}}=\left(J_{i}^{-1}\right)_{Q_{i}}$ for all $i$. We now have two cases to consider. Suppose first that $f$ is not a $p$ th power modulo $p^{2} S$. We will show that $R$ is Cohen-Macaulay. By our discussion in section two, $Q_{1}, \ldots, Q_{t}$ are exactly the height one primes $Q \subseteq S[\omega]$ for which $S[\omega]_{Q}$ is not a DVR, so by Proposition 2.1 and Lemma 3.3, $R=J^{-1}$ for $J:=J_{1} \cap \cdots \cap J_{t}$. Set $B:=S[W]_{(W, N)}$ (for $N$, the maximal ideal of $S$ ) and use "tilde" to denote pre-images in $B$. By Lemma 3.4, $\tilde{J} \subseteq B$ is a grade two perfect ideal. Therefore, $p \cdot d \cdot \cdot_{B}(J)=p \cdot d \cdot{ }_{B}\left(J^{-1}\right)=1$, by Proposition 2.1(iii). Thus, $\operatorname{depth}_{B}\left(J^{-1}\right)=\operatorname{dim}(B)-1$, so $\operatorname{depth}_{S}(R)=\operatorname{dim}(R)$, which is what we want.

Suppose that $f$ is a $p$ th power modulo $p^{2} S$. Write $f=h^{p}+p^{2} g$, for $h, g \in S$, $p \nmid h$. Then $P=(\omega-h, p)$. Moreover, $P$ and $Q_{1}, \ldots, Q_{t}$ are the height one primes $Q \subseteq S[\omega]$ for which $S[\omega]_{Q}$ is not a DVR. By Proposition 2.1 and Lemma 3.2, $R=J^{-1}$, for $J:=J_{1} \cap \cdots \cap J_{t} \cap P$. Now, as in the proof of Lemma 3.3, $J_{i}^{-1}$ is generated as an $S[\omega]$-module by the set $\left\{1, \frac{\lambda_{i} a_{i}}{\omega^{s(i, 1)}}, \ldots, \frac{\lambda_{i} a_{i}^{e_{i}-1}}{\omega^{s\left(i, e_{i}-1\right)}}\right\}$, where, for each $i, \lambda_{i}:=\prod_{i \neq j=1}^{r} \lambda a_{j}^{e_{j}}$. Thus $K_{i}=\left(\omega^{p-s(i, 1)}, \omega^{p-s(i, 2)} a_{i}, \ldots, a_{i}^{e_{i}-1}\right) S[\omega]$, for $K_{i}:=a_{i}^{e_{i}-1} \cdot J_{i}^{-1}$ and $1 \leq i \leq t$. By Lemma 3.3, $K_{i} \subseteq J_{i}$, so upon setting $I:=K_{1} \cap \cdots \cap K_{t} \cap P$, it follows from Lemma 3.5 that $I^{-1}$ is a $J^{-1}$-module (since this holds locally for every height one prime in $S[\omega])$. Taking $M:=I^{-1}$, we will show that $M$ is the required module. For this, we claim that $\tilde{I} \subseteq B$ is a grade two perfect ideal. If the claim holds, $1=p \cdot d \cdot B(I)=p \cdot d \cdot{ }_{B}\left(I^{-1}\right)=p \cdot d \cdot B(M)$. Thus $\operatorname{depth}_{B}(M)=\operatorname{dim}(B)-1$, so depth ${ }_{S}(M)=\operatorname{dim}(R)$, which is what we want.

To prove the claim, we set $\tilde{K}:=\tilde{K}_{1} \cap \cdots \cap \tilde{K}_{t}$ and consider the short exact sequence

$$
0 \longrightarrow B / \tilde{I} \longrightarrow B / \tilde{K} \oplus B / \tilde{P} \longrightarrow B /(\tilde{K}+\tilde{P}) \longrightarrow 0 .
$$

Since $\tilde{K}$ is a grade two perfect ideal (by Lemma 3.4), the Depth Lemma and the Auslander-Buchsbaum formula imply that $\tilde{I}$ is a grade two perfect ideal, once we $\operatorname{show} \operatorname{depth}(B /(\tilde{K}+\tilde{P}))=\operatorname{dim}(B)-3$. Set $a:=a_{1}^{e_{1}-1} \cdots a_{t}^{e_{t}-1}$. We now argue that $\tilde{K}+\tilde{P}=(a, p, W-h)$. If we can show this, clearly $\operatorname{depth}(B /(\tilde{K}+\tilde{P}))=\operatorname{dim}(B)-3$ and we will have verified the claim. Take $\tilde{k} \in \tilde{K}$ and consider its image $k$ in $K \subseteq S[\omega]$. Select $Q \subseteq S[\omega]$, a height one prime. If $Q=Q_{i}$, for some $1 \leq i \leq t$, then $k \in\left(a_{i}^{e_{i}-1} J_{i}^{-1}\right)_{Q_{i}}=a R_{Q_{i}}$. If $Q \neq Q_{i}$ for any $1 \leq i \leq t$, then clearly $k \in a R_{Q}=R_{Q}$. It follows that $k \in a R \cap S[\omega]$. In other words, $k$ is integral over the principal ideal $a S[\omega]$. Therefore, the image of $k$ in $S[\omega] /(\omega-h, p)=S / p S$ is integral over the principal ideal generated by the image of $a$. Since $S / p S$ is integrally closed, the image of $k$ in $S / p S$ is a multiple of the image of $a$. Therefore, $\tilde{k} \in(a, p, W-h)$ in $S[W]$. It follows that $\tilde{K} \subseteq(a, p, W-h)$. Since $a \in \tilde{K}$, we obtain $\tilde{K}+\tilde{P}=(a, p, W-h)$, which is what we want. This completes the proof of Theorem 3.8.

Remark 3.9. Of course if $S$ is an unramified regular local ring, $S$ fulfills our standing hypotheses, so Theorem 3.8 applies. However, the theorem also applies to certain ramified regular local rings. For instance, take $T$ to be the ring $\mathbb{Z}\left[X_{1}, \ldots, X_{d}\right]$ localized at $\left(p, X_{1}, \ldots, X_{d}\right)$ and let $H \in \mathbb{Z}\left[X_{1}, \ldots, X_{d}\right]$ be any polynomial in $\left(X_{1}, \ldots, X_{d}\right)^{2}$ for which $\mathbb{Z}_{p}\left[X_{1}, \ldots, X_{d}\right] /(\bar{H})$ is an integrally closed domain. If we set $S:=T /(p-H)$, then $S$ is a ramified regular local ring and $S / p S$ is an integrally closed domain. 
We close with an example where $R$ is not a free $S$-module, yet $R$ admits a finite, birational module which is a free $S$-module. The example is an unramified variation of Koh's Example (2.4).

Example 3.10. Let $S$ be an unramified regular local ring having mixed characteristic 3 and take $x, y \in S$ such that $3, x, y$ form part of a regular system of parameters. Set $a:=x y^{4}+9, b:=x^{4} y+9$ and $f:=a b^{2}$, so $\omega^{3}=f=a b^{2}=h^{3}+9 g$, for $h=x^{3} y^{2}$. From Lemmas 3.2 and 3.3 it follows that $R=(Q \cap P)^{-1}$ for $Q:=(\omega, b)$ and $P:=(\omega-h, 3)$. Set $J:=Q \cap P$. We first show that $R=J^{-1}$ is not a free $S$-module. Suppose to the contrary that $J^{-1}$ is free over $S$. As in the proof of Theorem 3.8, set $B:=S[W]_{(N, W)}$ and use "tilde" to denote pre-images in $B$. Since $J^{-1}$ is free over $S$, we have p.d. $B\left(J^{-1}\right)=1$, so $J^{-1}$ is a grade one perfect $B$-module. By [KU, Proposition 3.6], $J$ is a grade one perfect $B$-module, so $\tilde{J}$ is a grade two perfect ideal. On the other hand, $\operatorname{depth}_{B}(B / \tilde{J})=1+\operatorname{depth}_{B}(B /(\tilde{Q}+\tilde{P}))$. But, $\tilde{Q}+\tilde{P}=\left(W, x^{4} y, x^{3} y^{2}, 3\right) B$, so $B /(\tilde{Q}+\tilde{P})=S /\left(3, x^{4} y, x^{3} y^{2}\right) S$, which is easily seen to have depth equal to $\operatorname{depth}(S)-3=\operatorname{depth}(B)-4$. This is a contradiction, so it must hold that $R$ is not a free $S$-module.

Now, $Q^{-1}$ is generated as an $S[\omega]$-module by $\left\{1, \frac{a b}{\omega}\right\}$. If we set $K:=b \cdot Q^{-1}$, then $K=\left(\omega^{2}, b\right) S[\omega]$. The proof of Theorem 3.8 shows that $M:=(K \cap P)^{-1}$ is a finite, birational $R$-module satisfying $\operatorname{depth}_{S}(M)=\operatorname{dim}(R)$. In other words, $M$ is an $R$-module which is free over $S$. To calculate a basis for $M$, one must calculate $K \cap P$ and then use Proposition 2.1. We leave it to the reader to check that $K \cap P=\left(\omega^{2}-h^{2}-9 x^{2} y^{3}, b(\omega-h), 3 b\right)$. Therefore, $K \cap P=I_{2}(\phi)$ for

$$
\phi=\left(\begin{array}{cc}
-b & 0 \\
\omega+h & -3 \\
-3 x^{2} y^{3} & \omega-h
\end{array}\right) .
$$

The augmented matrix that determines $(K \cap P)^{-1}=M$ is the $3 \times 3$ matrix

$$
\left(\begin{array}{ccc}
-b & 0 & \omega \\
\omega+h & -3 & x^{2} y^{3} \\
-3 x^{2} y^{3} & \omega-h & t
\end{array}\right)
$$

where $t$ is defined by the equation $x^{5} y^{5}=a b+3 t$. By Proposition 2.1, $M$ is generated as an $S[\omega]$-module by the set $\{1, \gamma, \delta\}$, for

$$
\gamma:=\frac{-3 t-x^{2} y^{3}(\omega-h)}{\omega^{2}-h^{2}-9 x^{2} y^{3}}=\frac{\omega}{b}, \quad \delta:=\frac{-b t+3 x^{2} y^{3} \omega}{b(\omega-h)}=\frac{\omega^{2}+\omega h+h^{2}+9 x^{2} y^{3}}{3 b} .
$$

If we show that $\{1, \gamma, \delta\}$ also generate $M$ as an $S$-module, then since they are clearly linearly independent over $S$, they form a basis for $M$ as an $S$-module. To see that $\{1, \gamma, \delta\}$ generate $M$ as an $S$-module, it suffices to show that $\omega, \omega \cdot \gamma$ and $\omega \cdot \delta$ can be expressed as $S$-linear combinations of $\{1, \gamma, \delta\}$. This clearly holds for $\omega$. Using $9 x^{2} y^{3}=b x^{2} y^{3}-x^{6} y^{4}$, we obtain

$$
\omega \cdot \gamma=\frac{\omega^{2}}{b}=-x^{2} y^{3} \cdot 1-h \cdot \gamma+3 \cdot \delta .
$$

Since $\omega^{3}=h^{3}+9 g$ and $g=x^{5} y^{5}+b x y^{4}+b^{2}$, we get

$$
\omega \cdot \delta=\left(3 x y^{4}+3 b\right) \cdot 1+3 x^{2} y^{3} \cdot \gamma+h \cdot \delta,
$$

and the example is complete. 


\section{ACKNOWLEDGEMENTS}

I would like to thank both the referee and Craig Huneke for their helpful comments on how to improve the readability of this paper.

\section{REFERENCES}

[EU] D. Eisenbud and B. Ulrich, Modules that are finite birational algebras, Illinois Jl. Math 141 No. 1 (1997), 10-15. CMP 97:08

$[\mathrm{H}] \quad$ M. Hochster, Topics in the homological theory of modules over commutative rings, C.B.M.S. Reg. Conf. Ser. in Math., vol. 24, A.M.S., Providence, RI, 1975. MR 51:8096

[HM] M. Hochster and J.E. McLaughlin, Splitting theorems for quadratic ring extensions, Illinois Jl. Math. 127 No. 1 (1983), 94-103. MR 85c:13015

[Kap] I. Kaplansky, Commutative Rings II, University of Chicago, Lecture Notes.

[Ka] D. Katz, $P^{n}$ th root extensions in mixed characteristic $p$, preprint (1997).

[KU] S. Kleiman and B. Ulrich, Gorenstein algebras, symmetric matrices, self-linked ideals, and symbolic powers, Trans. AMS 349 (1997), 4973-5000. MR 98c:13019

[Ko] J. Koh, Degree $p$ extensions of an unramified regular local ring of mixed characteristic $p>0$, J. of Algebra 99 (1986), 310-323. MR 87j:13027

[MP] D. Mond and R. Pellikaan, Fitting ideals and multiple points of analytic mappings, Springer Lecture Notes in Mathematics 1414 (1989), 107-161. MR 91e:32035

[R] P. Roberts, Abelian extensions of regular local rings, Proc AMS 78, No 3 (1980), 307-319. MR 81a: 13017

[V] W. Vasconcelos, Computing the integral closure of an affine domain, Proc. AMS 113 (1991), 633-638. MR 92b:13013

Department of Mathematics, University of Kansas, Lawrence, Kansas 66045

E-mail address: dlk@math.ukans.edu 